\title{
Numerical Simulations of Fluorescence Resonance Energy Transfer in Diblock Copolymer Lamellae
}

Jian Yang, Mitchell A. Winnik*

Department of Chemistry, University of Toronto, 80 St. George Street,

Toronto, Ontario, Canada M5S 3H6

Tadeusz Pakula

Max-Planck-Institute für Polymerforschung, D-55021 Mainz, Postfach 3148, Germany

\section{Supporting Information}

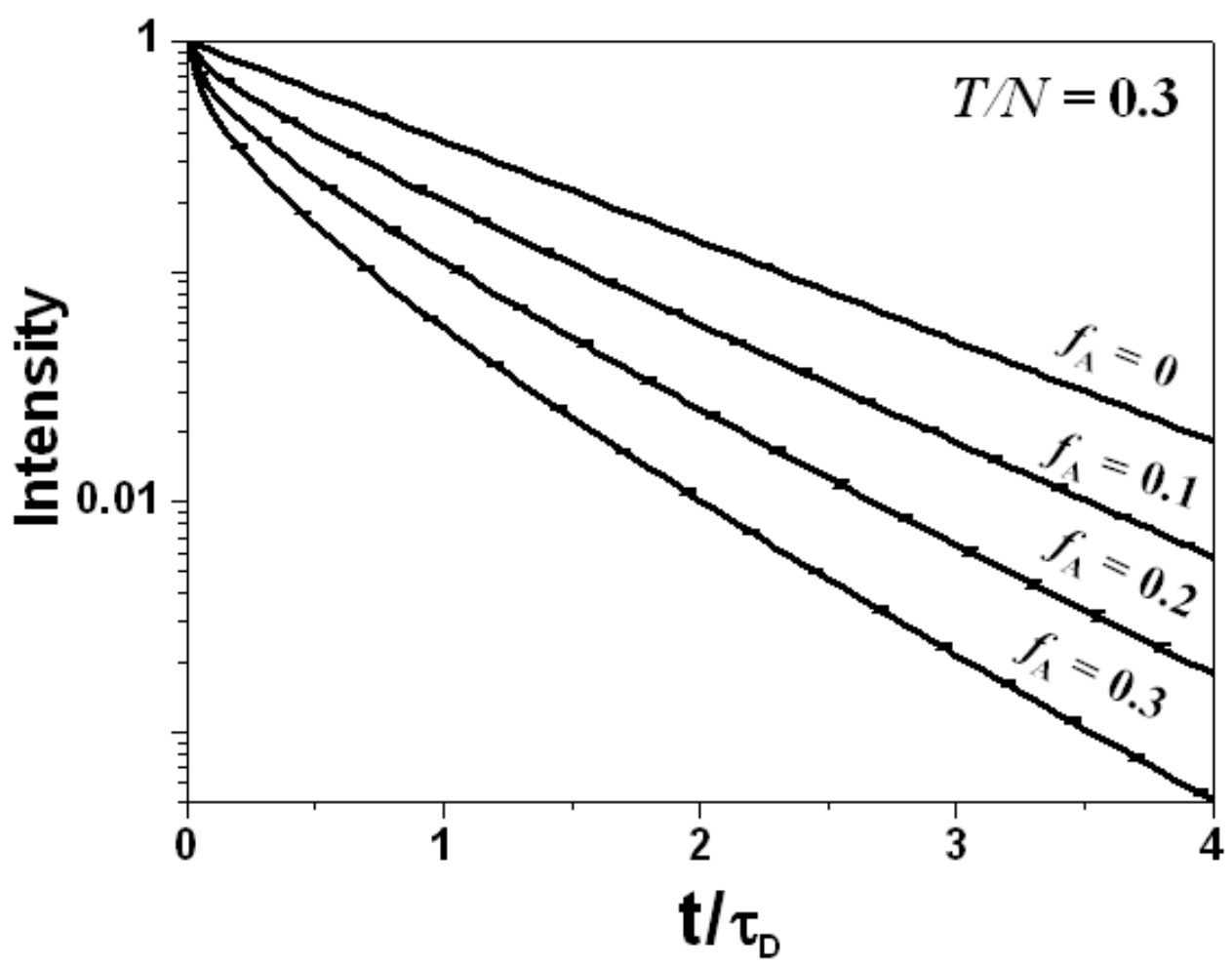

Figure S1. Delta-function fluorescence decays of the simulated system $(\mathrm{T} / \mathrm{N}=0.3)$ at different fractions of acceptor $\left(f_{\mathrm{A}}=\mathrm{N}_{\mathrm{A}} /\left(\mathrm{N}_{\mathrm{A}}+\mathrm{N}_{\mathrm{D}}\right)\right)$. We chose $\mathrm{R}_{0}=5 \mathrm{a}$ and the junction vector as the dipole orientation. $f_{\mathrm{A}}$ and $\mathrm{T} / \mathrm{N}$ values are indicated in the figures. The error bars are the standard deviation for 5 parallel random selections of the loci of donors and acceptors for the simulated system. 


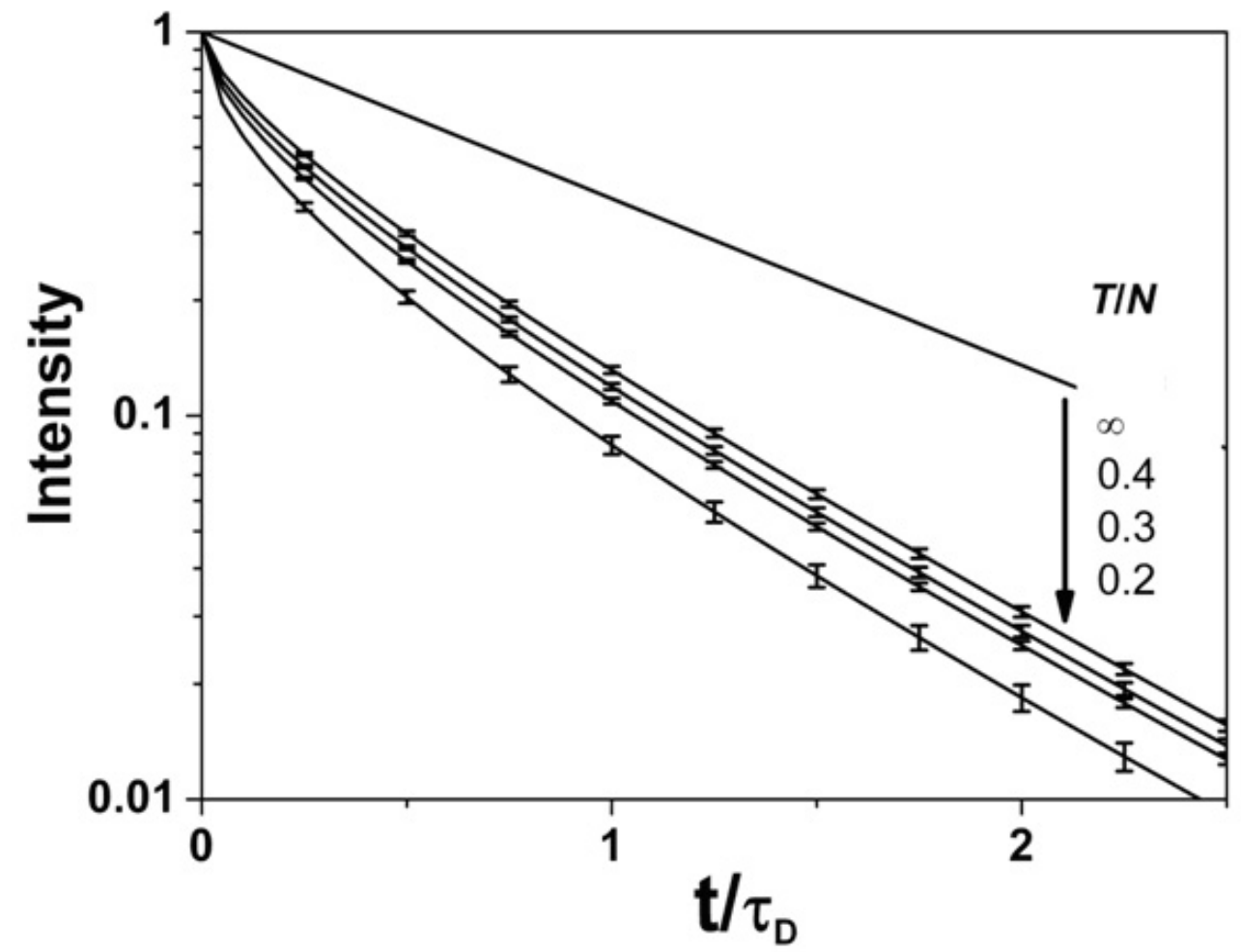

Figure S2. Delta-function fluorescence decays calculated using the junction vector as the dipole orientation for $f_{\mathrm{A}}=0.2$ and $\mathrm{R}_{0}=5 \mathrm{a}$ at the different temperatures indicated in the figure. The uppermost straight line is the unquenched fluoresce decay $\left(f_{\mathrm{A}}=0\right)$.

\section{FRET Kinetics Used for Interface Thickness Determination for Diblock Copolymer \\ Lamellae}

To obtain information about the interface thickness in the bulk state for block copolymers containing donor and acceptor dyes at the junction, we combined the Helfand - Tagami mean field model for the structure of diblock copolymers ${ }^{1}$ with the theory for FRET kinetics in lamellar structures. This theory is based on the Klafter and Blumen model for FRET in restricted geometries. ${ }^{2}$ A brief summary can be given as follows. The junction distribution density $P_{j}(z)$ in diblock copolymer lamella can be expressed as 


$$
P_{j}(z)=\frac{2 \pi}{\delta} \sec h\left(\frac{2 z}{\delta}\right)
$$

where $\delta$ is the interface thickness, and $z$ is the distance normal to the plane of the interface, taken to be centered at $z=0$. Eq S1 also represents the spatial distribution of junction-bound dye molecules across the interface in diblock copolymer lamella. FRET experiments take advantage of the distance-dependence of the energy transfer rate $w(r){ }^{3}$

$$
w(r)=\frac{3}{2} \kappa^{2} \frac{R_{0}^{6}}{\tau_{D} r^{6}}
$$

where $r$ is the distance between the centers of the transition dipoles of the donor and acceptor, and $\kappa^{2}$ is the orientation factor for the coupling of donor and acceptor dipoles. The Förster radius $R_{0}$ can be obtained from spectral overlap method by assuming $\kappa^{2}=2 / 3$, appropriate for the case of rapid dipole reorientation in the medium. In the theory of energy transfer in restricted geometries, donor groups are excited with equal probability independent of their location in the matrix. For each excited donor, the probability of FRET is described by integration over the distance dependence of $w(r)$ and the distribution of acceptors imposed by the geometry of the system. For donors and acceptors distributed along the z-axis normal to a dividing surface, the donor decay profile $I_{D}(t)$ can be expressed as ${ }^{2}$

$$
\begin{aligned}
& I_{D}(t)=I_{0} \exp \left(-t / \tau_{D}\right) \int C_{D}(z) \exp [-g(z, t)] d z \\
& g(z, t)=2 \pi \int_{o}^{\infty}\left\langle C_{A}(r, z)\right\rangle[1-\exp (-t w(r))] r d r \\
& \left\langle C_{A}(r, z)\right\rangle=N_{A} \int_{z-r}^{z+r} C_{A}\left(r^{\prime}\right) d r^{\prime}
\end{aligned}
$$


where $I_{0}$ is the intensity at $t=0 . C_{D}(z)$ and $C_{A}(r, z)$ describe the donor and acceptor distributions respectively. Since the donor and acceptor dyes are attached to the junctions of the block copolymers, their distribution follows that of the junctions, and

$$
C_{D}(z)=C_{D}^{0}(z) P_{j}(z) ; \quad C_{A}(z)=C_{A}^{0}(z) P_{j}(z)
$$

where $C_{D}^{0}(z)$ and $C_{A}^{0}(z)$ refer, respectively, to the bulk-averaged molar concentration of donor- and acceptor groups in the sample.
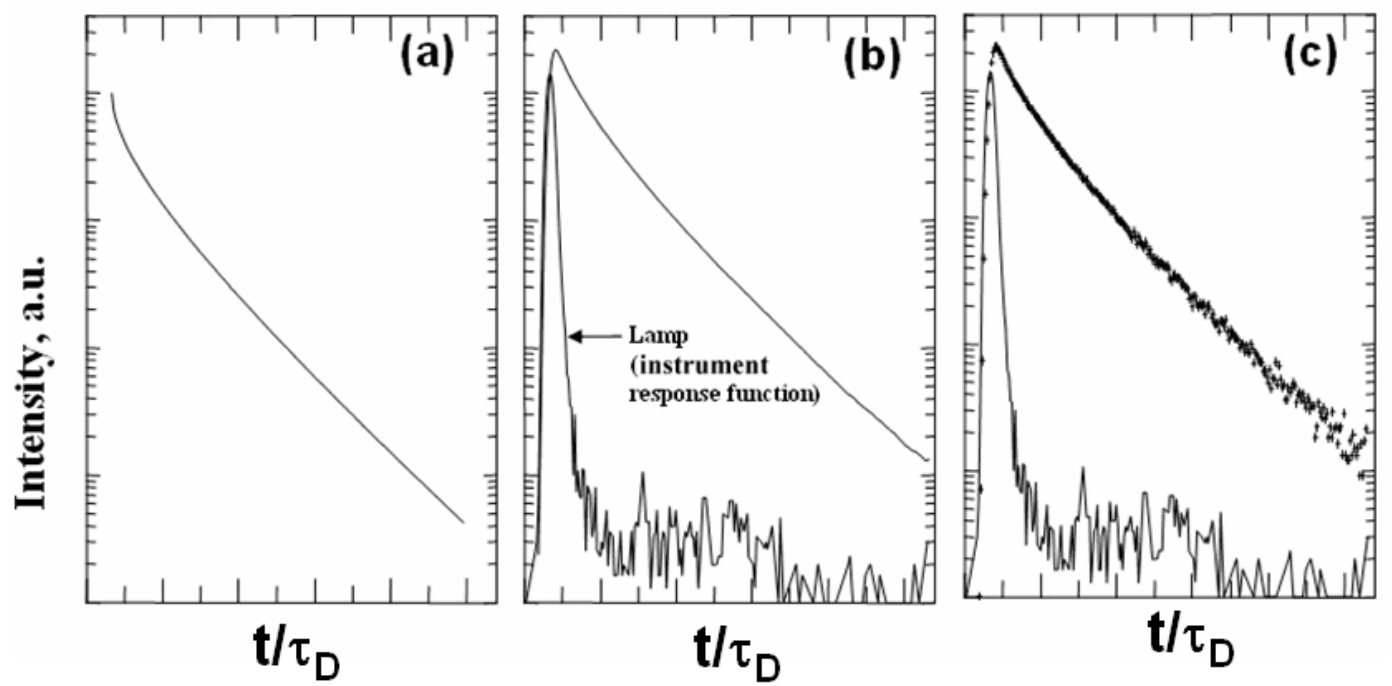

Figure S3 Procedure for the generation of simulated experimental fluorescence decay profiles. (a) delta function fluorescence decay. (b) experiment-like fluorescence decay obtained from convolution of the decay in (a) with the instrument response function, indicated in the figure. (c) Simulated experimental fluorescence decay obtained by adding Poisson noise at each channel of the experiment-like decay shown in (b). Here $t / \tau_{D}$ is dimensionless time, where $\tau_{\mathrm{D}}$ is the unquenched donor lifetime.

\section{Generation of Simulated Experimental Decay Profiles}

A procedure for the generation of simulated experimental fluorescence decay profiles is show in Figure S3. First we convoluted each delta-function fluorescence decay curve (Figure S3a, calculated as described in the previous section) with an instrument response function, as indicated in Figure S3b. Then Poisson noise was added at each 
channel to give the simulated experimental decay profile (Figure S3c). The instrument response function was obtained by measuring scattered light from a LUDOX ${ }^{\circledR}$ solution using a Single Photon Timing system. The timescale of the measured instrument response function is $1.04 \mathrm{~ns}$ per channel. We normalized the time scale to $0.05 \mathrm{t} / \tau_{\mathrm{D}}\left(\tau_{\mathrm{D}}\right.$ is the unquenched donor lifetime) to obtain a common dimensionless timescale for experimental and simulation data. The maximum number of counts per channel for the simulated experimental decays was set to 20,000. The delta-function fluorescence decays selected for the generation of the simulated experimental decays were calculated with $\mathrm{R}_{0}$ $=5 \mathrm{a}^{4}$ and a pre-averaged orientation factor $<|\kappa|>^{2}=0.476$. Since our FRET methodology cannot consider the individual orientation factors for each D-A pairs. A pre-averaged orientation factor (here we choose $\left\langle\left.|\kappa|\right|^{2}=0.476\right.$ ) has to be chosen in the calculation of energy transfer. In order to avoid any effect introduced by changing orientation factor on the data analysis, we set $\langle|\kappa|\rangle^{2}=0.476$ in the generation of simulated experimental decay profiles. In this way, interface obtained from the decay curve fitting contains no information from the selection of orientation factor.

\section{References and Note:}

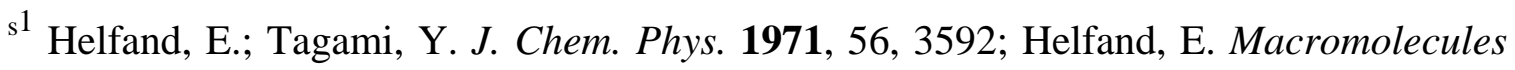
1975, 8, 552; Helfand, E.; Wasserman, Z. R. Macromolecules 1980, 13, 994.

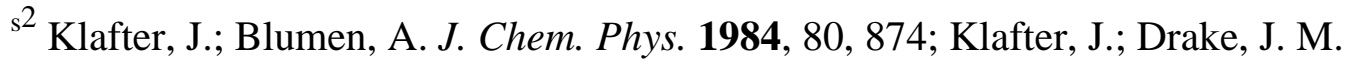
Molecular dynamics in restricted geometries, Wiley, New York, 1989; Drake, J. M.; Klafter, J.; Levitz, P. Science 1991, 251, 1574. 
${ }^{s 3}$ Lakowicz, J. R., Ed. Principles of Fluorescence Spectroscopy, Plenum Press: New York, 1999.

${ }^{{ }^{4}}$ Note that $\mathrm{R}_{0}$ the Förster radius is defined from the D-A spectral overlap integral along with the assumption that $\left\langle k^{2}\right\rangle=2 / 3$. 